\title{
Fractonic View of Folding and Tearing Paper: Elasticity of Plates Is Dual to a Gauge Theory with Vector Charges
}

\author{
Nandagopal Manoj, ${ }^{1, *}$ Roderich Moessner, ${ }^{2, \dagger}$ and Vijay B. Shenoy ${ }^{1, \$}$ \\ ${ }^{1}$ Centre for Condensed Matter Theory, Department of Physics, Indian Institute of Science, Bangalore 560012, India \\ ${ }^{2}$ Max-Planck-Institut für Physik komplexer Systeme, Dresden 01187, Germany
}

(Received 12 January 2021; accepted 1 July 2021; published 4 August 2021)

\begin{abstract}
We offer a fractonic perspective on a familiar observation - a flat sheet of paper can be folded only along a straight line if one wants to avoid the creation of additional creases or tears. Our core underlying technical result is the establishment of a duality between the theory of elastic plates and a fractonic gauge theory with a second rank symmetric electric field tensor, a scalar magnetic field, a vector charge, and a symmetric tensor current. Bending moment and momentum of the plate are dual to the electric and magnetic fields, respectively. While the flexural waves correspond to the quadratically dispersing photon of the gauge theory, a fold defect is dual to its vector charge. Crucially, the fractonic condition constrains the latter to move only along its direction, i.e., the fold's growth direction. By contrast, fracton motion in the perpendicular direction amounts to tearing the paper.
\end{abstract}

DOI: 10.1103/PhysRevLett.127.067601

Introduction.-Gauge theories play an eminent role in physics, all the way from elementary particle theory at high energies to the emergent descriptions of topological quantum matter at low energies. The latter include instances such as the toric code [1], which has an important role in the context of topologically protected quantum computation.

It is thus natural to look for generalizations of the gauge theories familiar from these settings. In this vein, fracton phases [2-11] are perhaps the latest entrant. The most salient of their novel properties is the appearance of the eponymous fracton particles, which exhibit restricted, or "fractional" mobility, see, e.g., the recent reviews in Refs. $[12,13])$. It is those charges, but not their corresponding point dipoles, which are subject to fractonic mobility restrictions [14,15]. In addition, these tensor gauge theories also support gapless "photons" much like the usual electromagnetism. Such theories have also found generalizations to include extended fractons (line, surface like excitations) and varied dispersions of the gapless modes [16,17].

Reference [18] demonstrated that a second rank tensor scalar charge fractonic gauge theory is dual to the theory of crystalline elastic solids in two spatial dimensions, with fracton charges dual to immobile disclinations, while their dipoles are dual to dislocations which are subject to lesser mobility restrictions. The gauge structure, viewed from the perspective of the elastic solid, arises from the fact the dynamical equation of motion of the elastic solid written in terms of the stress tensor and the momentum density can be resolved by writing stress tensor and momentum density using symmetric tensor gauge fields (similar ideas are also found in earlier literature, cf. Refs. [19-22]). These developments have led to related explorations [16,23-29].
The present Letter likewise identifies a "real life" example of a physical system that is dual to a fractonic gauge theory with vector charges introduced in Refs. [30,31] (see also Refs. [14,15]). This gauge theory is formulated in terms of a symmetric second rank tensor electric field and a scalar magnetic field. We show that such a theory is dual to the theory of elastic plates [32,33]. Our duality, summarized in Table I, maps the plate bending moment to the electric field of the gauge theory, the plate momentum density to the magnetic field, and the flexural wave in the plate to the photon of the gauge theory. Crucially, "fold defects" of the plate are mapped to the fractonic vector charges of the gauge theory. This provides a fractonic perspective of the observation that a flat sheet of paper can be folded only along a straight line keeping the rest of the paper crease or tear free: the "end point of a fold" is a vector fractonic charge that can move only along the direction of the fold. Conversely, tearing of the paper can be understood as motion of a fractonic vector charge that violates the fractonic condition, that leaves behind a trail of "tear defects." The gauge theory supports a photon which

TABLE I. Duality correspondence.

\begin{tabular}{lc}
\hline \hline Theory of plates with defects & Fractonic vector charge theory \\
\hline Bending moment $\left(M_{\alpha \beta}\right)$ & Electric field $\left(\epsilon_{\alpha \gamma} \epsilon_{\beta \delta} E_{\gamma \delta}\right)$ \\
Momentum density $(P)$ & Magnetic field $(B)$ \\
Fold density $\left(\zeta_{\alpha}\right)$ & Charge density $\left(\epsilon_{\beta \alpha} \rho_{\beta}\right)$ \\
Velocity curvature - Curvature & Current density $\left(J_{\alpha \beta}\right)$ \\
$\quad$ velocity $\left[\epsilon_{\alpha \delta} \epsilon_{\beta \gamma}\left(V_{\gamma \delta}^{d}-\partial_{t} R_{\gamma \delta}^{d}\right)\right]$ & Photon \\
Flexural wave & \\
\hline \hline
\end{tabular}


disperses as $\omega$ (frequency) $\sim|k|^{2}$ (wavevector). This corresponds to the flexural wave of the elastic plate whichunlike the phonon-has a quadratic dispersion.

Vector charge fracton theory.-Consider a symmetric second rank electric field tensor $E_{\alpha \beta}$, and a scalar magnetic field $B$ in two dimensions, with position vector $x=\left(x_{1}, x_{2}\right)$, and time $t$. Central to our discussion is a vector charge $\rho_{\alpha}(x, t)(\alpha=1,2) \quad[14,15,30,31,34]$. The form of Gauss's law which will endow the charge with fractonic character reads $\partial_{\beta} E_{\beta \alpha}=\rho_{\alpha}$ (see below) where repeated Greek (spatial) indices are summed over, and $\partial_{\alpha}$ is the derivative with respect to $x_{\alpha}$.

Electric and magnetic fields are encoded by a set of gauge fields $\left(\phi_{\alpha}, A_{\alpha \beta}\right)$, with the vector field $\phi_{\alpha}(x, t)$ the analog of the scalar potential and the symmetric second rank tensor $A_{\alpha \beta}(x, t)$ that of the vector potential of Maxwell electromagnetism, via

$E_{\alpha \beta}=-\frac{1}{2}\left(\partial_{\alpha} \phi_{\beta}+\partial_{\beta} \phi_{\alpha}\right)-\partial_{t} A_{\alpha \beta}, \quad B=\epsilon_{\alpha \gamma} \epsilon_{\beta \delta} \partial_{\gamma} \partial_{\delta} A_{\alpha \beta}$,

invariant under the gauge transformation induced by $f_{\alpha}(x, t)$ :

$\phi_{\alpha} \rightarrow \phi_{\alpha}+\partial_{t} f_{\alpha}, \quad A_{\alpha \beta} \rightarrow A_{\alpha \beta}-\frac{1}{2}\left(\partial_{\alpha} f_{\beta}+\partial_{\beta} f_{\alpha}\right)$.

The complete theory is described by a Lagrangian density

$$
L=\frac{1}{2} \kappa_{\alpha \beta \gamma \delta} E_{\alpha \beta} E_{\gamma \delta}-\frac{1}{2 \mu} B^{2}-\rho_{\alpha} \phi_{\alpha}+J_{\alpha \beta} A_{\alpha \beta}
$$

with dielectric tensor $\kappa_{\alpha \beta \gamma \delta}$ and magnetic permeability $\mu$, with $\kappa_{\alpha \beta \gamma \delta}=\kappa_{\beta \alpha \gamma \delta}=\kappa_{\alpha \beta \delta \gamma}=\kappa_{\gamma \delta \alpha \beta} . J_{\alpha \beta}$ is the symmetric second rank current tensor. Gauge invariance under Eq. (2) implies

$$
\partial_{t} \rho_{\beta}+\partial_{\alpha} J_{\alpha \beta}=0 .
$$

The principle of least action provides two Maxwell equations

$$
\begin{gathered}
\partial_{\alpha} \kappa_{\alpha \beta \gamma \delta} E_{\gamma \delta}=\rho_{\beta} \\
\partial_{t} \kappa_{\alpha \beta \gamma \delta} E_{\gamma \delta}-\frac{1}{\mu} \epsilon_{\alpha \gamma} \epsilon_{\beta \delta} \partial_{\gamma} \partial_{\delta} B+J_{\alpha \beta}=0 .
\end{gathered}
$$

From Eq. (1), the vector charge version of Faraday law reads

$$
\epsilon_{\alpha \gamma} \epsilon_{\beta \delta} \partial_{\gamma} \partial_{\delta} E_{\alpha \beta}+\partial_{t} B=0 .
$$

The fractonic character of the vector charge is revealed as follows. For a system with area $S$, Eq. (4) implies conservation of the total charge $\int_{S} d^{2} x \rho_{\alpha}$. Now, there is an additional conserved quantity $\Sigma$ the moment of the vector charge

$$
\Sigma=\int_{S} d^{2} x \epsilon_{\alpha \beta} x_{\alpha} \rho_{\beta}
$$

Indeed, the time derivative of $\Sigma$ vanishes owing to the divergence theorem and the symmetric nature of the current $J_{\alpha \beta}$. The consequence of this conservation law is that an isolated point charge has fractonic character-it is in fact a lineon [35] as it can only move along its own vector $Q_{\alpha}$. This can be illustrated by a point vector charge $\rho_{\alpha}(x)=$ $Q_{\alpha} \delta^{(2)}\left(x-x^{0}\right)$ located at $x^{0}\left(\delta^{(2)}\right.$ denotes the two dimensional Dirac delta function). The conservation law Eq. (8) imposes that any change of $x^{0}$ must obey $x_{\alpha}^{0} \rightarrow x_{\alpha}^{0}+s Q_{\alpha}$ with $s \in \mathbb{R}$, to keep $\Sigma$ constant.

The theory supports a scalar, quadratically dispersing photon. For an isotropic system with ( $\delta_{\alpha \beta}$ is the Kronecker delta)

$$
\kappa_{\alpha \beta \gamma \delta}=\kappa_{1} \delta_{\alpha \beta} \delta_{\gamma \delta}+\frac{\kappa_{2}}{2}\left(\delta_{\alpha \gamma} \delta_{\beta \delta}+\delta_{\alpha \delta} \delta_{\beta \gamma}\right),
$$

$\omega(k)=\sqrt{\left\{\left(\kappa_{1}+\kappa_{2}\right) /\left[\kappa_{2}\left(2 \kappa_{1}+\kappa_{2}\right) \mu\right]\right\}}|k|^{2}$ carrying fields $B(x, t)=$ $B_{0} e^{\mathrm{i}(k x-\omega(k) t)}$ and $E_{\alpha \beta}(x, t)=\left(B_{0} / \mathrm{i}\right) \sqrt{\left\{\left(\kappa_{1}+\kappa_{2}\right) /\left[\kappa_{2}\left(2 \kappa_{1}+\kappa_{2}\right) \mu\right]\right\}} \times$ $\left\{\delta_{\alpha \beta}-\left[\left(2 \kappa_{1}+\kappa_{2}\right) /\left(\kappa_{1}+\kappa_{2}\right)\right] \hat{k}_{\alpha} \hat{k}_{\beta}\right\} e^{i[k x-\omega(k) t]}$ (with $\left.\stackrel{0}{i}=\sqrt{-1}\right)$.

Elastic plates. -The physics of a three-dimensional elastic solid [32] is described by a displacement field $u_{i}(r, t)$, a symmetric strain tensor field $\epsilon_{i j}(r, t)$ and a symmetric stress tensor field $\sigma_{i j}(r, t)$, where $r$ is a position vector of a material particle in three dimensions (latin indices $i, j$, etc., run over all spatial coordinates, $i=1,2,3)$. The strain tensor $\epsilon_{i j}=\frac{1}{2}\left(\partial_{i} u_{j}+\partial_{j} u_{i}\right)$ and the stress tensor are related by an elastic constitutive relation $\sigma_{i j}=C_{i j k l} \epsilon_{k l}$, where the elastic tensor $C_{i j k l}$ obeys $C_{i j k l}=C_{j i k l}=C_{i j l k}=$ $C_{k l i j}$. For isotropic solids, which is our focus here, $C_{i j k l}=$ $(E / 1+\nu)\left\{[\nu /(1-2 \nu)] \delta_{i j} \delta_{k l}+\frac{1}{2}\left(\delta_{i k} \delta_{j l}+\delta_{i l} \delta_{j k}\right)\right\}$, where $E$ and $\nu$ are, respectively, Young's modulus and Poisson's ratio. The Lagrangian density of the system is given by

$$
L=\frac{1}{2} \lambda \partial_{t} u_{i} \partial_{t} u_{i}-\frac{1}{2} C_{i j k l} \epsilon_{i j} \epsilon_{k l}
$$

with $\lambda$ the mass density of the solid. For a "slender" body (see the plate in Fig. 1) with thickness $h$ much smaller than its lateral dimensions, $L_{\alpha}$, an effective low energy theoryplate theory-that becomes increasingly accurate as $h / L_{\alpha} \rightarrow 0$ can be developed. This Ansatz uses a coordinate system $\left(r_{1}, r_{2}, r_{3}\right)=\left(x_{1}, x_{2}, z\right) \equiv(x, z)$, where $z$ is the coordinate normal to the plane (henceforth called the vertical). It uses the midplane displacement normal to itself, 


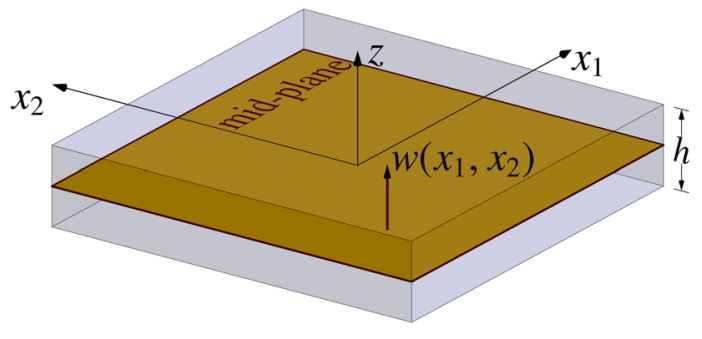

FIG. 1. Schematic of an elastic plate of thickness $h$. The deformation of the plate is described by the vertical (along the $z$ direction) displacement $w\left(x_{1}, x_{2}\right)$ of the midplane [Eq. (11)].

denoted by $w(x)=u_{3}(x, 0)$ to encode the full dynamics of the three-dimensional plate via $[32,33]$

$$
u_{1}(x, z)=-z \partial_{1} w, \quad u_{2}(x, z)=-z \partial_{2} w .
$$

In addition, the stress component $\sigma_{33}$ vanishes everywhere. With these given, we have $\sigma_{\alpha \beta}=\bar{C}_{\alpha \beta \gamma \delta} \epsilon_{\alpha \beta}$, where $\bar{C}_{\alpha \beta \gamma \delta}=$ $\left[E /\left(1-\nu^{2}\right)\right]\left[(1-\nu) \delta_{\alpha \beta} \delta_{\gamma \delta}+(\nu / 2)\left(\delta_{\alpha \gamma} \delta_{\beta \delta}+\delta_{\alpha \delta} \delta_{\beta \gamma}\right)\right]$. The effective Lagrangian density on the midplane reads

$$
L=\frac{\lambda_{p}}{2} \partial_{t} w \partial_{t} w-\frac{1}{2} D_{\alpha \beta \gamma \delta}\left(\partial_{\alpha} \partial_{\beta} w\right)\left(\partial_{\gamma} \partial_{\delta} w\right),
$$

where $\lambda_{p}=\lambda h$ is the mass per unit area of the plate, and

$$
D_{\alpha \beta \gamma \delta}=D\left[\nu \delta_{\alpha \beta} \delta_{\gamma \delta}+\frac{(1-\nu)}{2}\left(\delta_{\alpha \gamma} \delta_{\beta \delta}+\delta_{\alpha \delta} \delta_{\beta \gamma}\right)\right] \text {, }
$$

the bending modulus tensor, with $D=E h^{3} /\left[12\left(1-\nu^{2}\right)\right]$ the bending modulus. The quantity $\partial_{\alpha} \partial_{\beta} w\left(\equiv R_{\alpha \beta}\right)$ is the curvature tensor induced by the deformation $w$. The bending modulus obtains the bending moment from the curvature via $M_{\alpha \beta}=D_{\alpha \beta \gamma \delta} \partial_{\gamma} \partial_{\delta} w$. The plate supports "flexural waves," $w(x, t)=w_{0} e^{\mathrm{i}(k x-\omega(k) t)}$ which disperse as $\omega(k)=\sqrt{D / \lambda_{p}}|k|^{2}$.

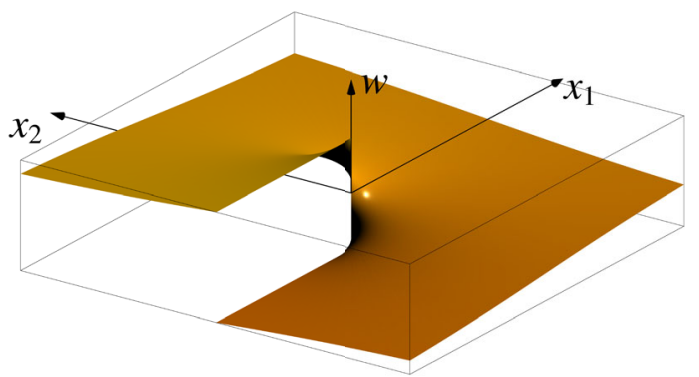

(a) Tear defect
The usual discussion of the theory of plates considers smooth single valued displacement fields $w(x, t)$. Here we discuss the types of defects that arise in the theory of plates. For this, we introduce an additional quantity, $\theta_{\alpha}=\partial_{\alpha} w$, the "slope of the deformed midplane" (see Fig. 1), or equivalently the rotation of material fiber vertical to the midplane.

Tear defect.-First, a tear defect of strength $a$ located at a point $x^{0}$, implies for a closed contour $C$ (which avoids the point $x^{0}$ ),

$$
\oint_{C} d x_{\alpha} \theta_{\alpha}=\left\{\begin{array}{ll}
a & \text { if } C \text { encloses } x^{0} \\
0 & \text { if } C \text { does not enclose } x^{0}
\end{array} .\right.
$$

Figure 2(a) shows an example of an isolated tear defect. This illustrates the multivalued nature of the displacement field $w$ resulting in the tear of the plate. The multivalued $w$ field is such that both the slope field and the curvature fields are smooth everywhere except at the location of the defect where they diverge. We define a density of such tear defects

$$
\int_{A} d^{2} x \tau=\oint_{C} d x_{\alpha} \theta_{\alpha}=\llbracket w \rrbracket_{C}
$$

with $A$ the area enclosed by $C$, and $\llbracket w \rrbracket_{C}$ the jump in the displacement field obtained upon traversing the contour $C$ and

$$
\tau=\epsilon_{\alpha \beta} \partial_{\alpha} \theta_{\beta} .
$$

For the single tear defect located at $x^{0}$ discussed above $\tau(x)=a \delta^{(2)}\left(x-x^{0}\right)$ [see Fig. 2(a) where $a=1$ and $x^{0}$ is the origin].

Fold defect.-Second, a fold defect [Fig. 2(b)] of strength $\psi_{\alpha}$ located at $x^{0}$ produces

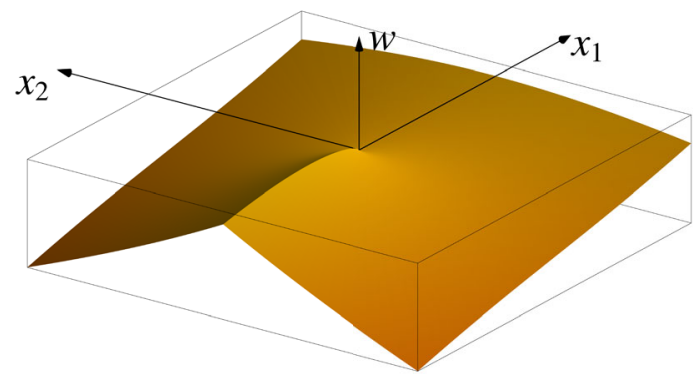

(b) Fold defect

FIG. 2. (a) Tear defect located at the origin with $w\left(x_{1}, w_{2}\right)=(1 / 2 \pi) \arctan \left(x_{2} / x_{1}\right)$ and tear defect density (eqn. (16)) $\tau=\delta^{(2)}(x)$ that satisfies Eq. (14) with $a=1$. (b) Fold defect located at the origin with $w\left(x_{1}, x_{2}\right)=(1 / 2 \pi)\left[x_{1} \ln (|x|)-x_{2}\right.$ arctan $\left.\left(x_{2} / x_{1}\right)\right]$ and defect density $\zeta_{\alpha}=-\delta_{\alpha 2} \delta^{(2)}(x)$ that satisfies Eq. (17) with $\psi_{\alpha}=-\delta_{\alpha 2}$. For the tear defect (a) the slopes $\theta_{\alpha}=\partial_{\alpha} w$ and curvatures $R_{\alpha \beta}=\partial_{\alpha} \partial_{\beta} w$ are smooth everywhere (except the origin), while for fold defect (b) only the latter applies. Function arctan returns a value in $[-\pi, \pi]$, with $\lim _{\varepsilon \rightarrow 0} \arctan (-\epsilon /-1)=-\pi$. 


$$
\oint_{C} d x_{\alpha} R_{\alpha \beta}=\left\{\begin{array}{ll}
\psi_{\beta} & \text { if } C \text { encloses } x^{0} \\
0 & \text { if } C \text { does not enclose } x^{0}
\end{array} .\right.
$$

Its curvature tensor is smooth everywhere except at the location of the defect. Further, the defect goes along with a fold in the plate, which terminates at the defect. For a continuous distribution of fold defects, the fold density $\zeta_{\alpha}$ is given by

$$
\oint_{C} d x_{\alpha} R_{\alpha \beta}=\int_{A} d^{2} x \zeta_{\alpha}=\llbracket \theta_{\alpha} \rrbracket_{C},
$$

where $\llbracket \theta_{\alpha} \rrbracket_{C}$ is the net jump in the slope when traversing the closed contour $C$ that encloses the area $A$. We thus get

$$
\zeta_{\alpha}=\epsilon_{\beta \gamma} \partial_{\beta} R_{\gamma \alpha}
$$

Again, for the fold defect shown in Fig. 2(b), $\zeta_{\alpha}=-\delta_{\alpha 2} \delta^{(2)}(x)$.

An important point to be noted is that for the given defect configuration, there are many distinct displacement fields $w^{d}(x)$ that will satisfy conditions of the type Eqs. (15) and (18). For example, the tear defect $\tau=a \delta^{(2)}(x)$ is equally well described by a different displacement field than that shown in Fig. 2(a) by a different choice of the branch of the arctan function. This situation is akin to the description of a superfluid vortex, or a magnetic monopole, and suggests the presence of an underlying gauge structure in the theory [19].

We now write the displacement $w$ as

$$
w(x, t)=w^{s}(x, t)+w^{d}(x, t),
$$

where $w^{s}(x, t)$ is the smooth or single-valued part of the deformation and $w^{d}(x, t)$ is a multivalued field that describes the defects, and insert this into the Lagrangian density [Eq. (12)].

Duality.-To establish the duality between plate and fracton theories, we introduce two Hubbard-Stratonovich fields $P(x, t)$ (momentum density) and $M_{\alpha \beta}(x, t)$ (bending moment) to treat the kinetic and potential energy terms in the Lagrangian Eq. (12):

$$
\begin{aligned}
\mathcal{S}\left[w, P, M_{\alpha \beta}\right]= & \int d t d^{2} x\left[\frac{1}{2} D_{\alpha \beta \gamma \delta}^{-1} M_{\alpha \beta} M_{\gamma \delta}-\frac{1}{2 \lambda_{p}} P^{2}\right. \\
& +P\left(\partial_{t} w^{s}+\partial_{t} w^{d}\right) \\
& \left.-M_{\alpha \beta}\left(\partial_{\alpha} \partial_{\beta} w^{s}+\partial_{\alpha} \partial_{\beta} w^{d}\right)\right]
\end{aligned}
$$

Integrating out the smooth displacement field $w^{s}$ gives

$$
\partial_{t} P+\partial_{\alpha} \partial_{\beta} M_{\alpha \beta}=0,
$$

which embodies momentum conservation (perpendicular to the mid-plane). We can now identify

$B(x, t) \equiv P(x, t), \quad \epsilon_{\alpha \gamma} \epsilon_{\beta \delta} E_{\gamma \delta}(x, t) \equiv M_{\alpha \beta}(x, t)$.

This shows that Eq. (22) can be resolved (identically satisfied) if $B$ and $E_{\alpha \beta}$ are expressed through the gauge fields $\phi_{\alpha}$ and $A_{\alpha \beta}$ as in Eq. (1). Using Eq. (23) and Eq. (1), the action Eq. (21) in terms of the gauge fields reads

$$
\begin{aligned}
\mathcal{S} & {\left[w^{d}, \phi_{\alpha}, A_{\alpha \beta}\right] } \\
= & \int d t d^{2} x\left[\frac{1}{2} \kappa_{\alpha \beta \gamma \delta} E_{\alpha \beta} E_{\gamma \delta}-\frac{1}{2 \mu} B^{2}+\left(\epsilon_{\alpha \delta} \epsilon_{\beta \gamma} \partial_{\alpha} \partial_{\beta} A_{\gamma \delta}\right) \partial_{t} w^{d}\right. \\
& \left.+\epsilon_{\alpha \gamma} \epsilon_{\beta \delta}\left(\frac{1}{2}\left(\partial_{\gamma} \phi_{\delta}+\partial_{\delta} \phi_{\gamma}\right)+\partial_{t} A_{\gamma \delta}\right) \partial_{\alpha} \partial_{\beta} w^{d}\right]
\end{aligned}
$$

where, using Eq. (9), the following identifications are made $\kappa_{\alpha \beta \gamma \delta} \equiv D_{\alpha \beta \gamma \delta}^{-1}, \quad \kappa_{1} \equiv-\left[\nu / D\left(1-\nu^{2}\right)\right], \quad \kappa_{2} \equiv[1 / D(1-\nu)]$, $\mu \equiv \lambda_{p}$. Finally, the action Eq. (24), after suitable integration by parts of the last two terms, reduces exactly to the action governed by the Lagrangian density Eq. (3) of the vector charge fracton theory. The dual charges and currents of the fracton theory are

$$
\begin{gathered}
\rho_{\alpha} \equiv \epsilon_{\alpha \delta} \epsilon_{\beta \gamma} \partial_{\beta} R_{\gamma \delta}^{d}=\epsilon_{\alpha \delta} \zeta_{\delta}, \\
J_{\alpha \beta}=\epsilon_{\alpha \delta} \epsilon_{\beta \gamma}\left(V_{\gamma \delta}^{d}-\partial_{t} R_{\gamma \delta}^{d}\right),
\end{gathered}
$$

where we have used Eq. (19), and $R_{\alpha \beta}^{d}=\partial_{\alpha} \partial_{\beta} w^{d}, V_{\alpha \beta}^{d}=$ $\partial_{\alpha} \partial_{\beta}\left(\partial_{t} w^{d}\right)$ are, respectively, the defect curvature field and defect "velocity curvature" field. The duality is summarized in Table I. We note here the duality of the vector charge gauge theory to a scalar field was noted in Ref. [30], although the connection to the theory of plates was not discussed.

Discussion.-We first address the connection between the vector charges of the gauge theory and the fold defects of plates. Consider a fold on a plate that lies long the $x_{1}$ axis terminating at the origin Fig. 2(b), such that the normals to the plate just below and just above the negative $x_{1}$ axis are tilted by a small angle $\psi$, corresponding to $\zeta_{\alpha}=\psi \delta_{\alpha 2} \delta^{(2)}(x)$ indicating a jump $\psi$ in the 2-component of the slope of the plate when traversing across the negative $x_{1}$ axis. This corresponds to a linenonic vector charge, eqn. (25), of $\rho_{\alpha}=\psi \delta_{\alpha 1} \delta^{(2)}(x)$ i.e., a "charge along the $x_{1}$ direction" located at the origin. Following the discussion near Eq. (8) we see that this charge is allowed to move only along the $x_{1}$ axis. Viewed, again, from the perspective of plates, we see that a point fold defect can only move-and the fold extended-in a direction perpendicular to its strength (which is a vector). We thus obtain a fractonic perspective on the observation that a flat sheet of paper can 
be folded smoothly (without the creation of additional creases or tears) only along a straight line.

Then, what about the tear and lineons? The connection can be seen by noting two points: First, note that the tear defect, Eq. (16) in fact is a dipole of fold defects. For instance, the tear defect $\tau(x)=a \delta^{(2)}(x)$ arises from a fold pair with $\zeta_{\alpha}(x)=\psi \delta_{\alpha 2}\left[\delta^{(2)}\left(x+\varepsilon \hat{e}_{2}\right)-\delta^{(2)}(x)\right]$, where $\hat{e}_{2}$ is the unit vector along the $x_{2}$ direction, such that $\lim _{\varepsilon \rightarrow 0} \psi \epsilon=a$. More generally, a dipole of fold defects located at a point $x^{0}$ has $\zeta_{\alpha}(x)=$ $\lim _{\varepsilon \rightarrow 0} \psi_{\alpha} \varepsilon \hat{n}_{\beta} \partial_{\beta} \delta^{(2)}\left(x-x^{0}\right)=a_{\alpha} \hat{n}_{\beta} \partial_{\beta} \delta^{(2)}\left(x-x^{0}\right)$, where $\hat{n}_{\beta}$ is a unit vector. This results in $\left(\lim _{\varepsilon \rightarrow 0} \psi_{\beta} \varepsilon=a_{\beta}\right)$, $\rho_{\alpha}(x)=\epsilon_{\alpha \beta} a_{\beta} \hat{n}_{\gamma} \partial_{\gamma} \delta^{(2)}\left(x-x^{0}\right)$ which is a tear defect of strength of $a_{\gamma} \hat{n}_{\gamma}$. From Eq. (8), $\Sigma_{\text {dipole }}=a_{\gamma} \hat{n}_{\gamma} \approx \varepsilon \psi_{\alpha} \hat{n}_{\gamma}$, independent of $x^{0}$.

Second, consider the displacement of a fold defect $\zeta_{\alpha}=\psi_{\alpha} \delta^{(2)}\left(x-x_{0}\right)$ from $x_{0}$ to $x_{0}+\varepsilon \hat{n}$ along the unit vector $\hat{n}$. Using Eq. (8) we see that the moment $\Sigma$ changes by an amount $\varepsilon \psi_{\alpha} \hat{n}_{\alpha}$. The moment $\Sigma$ can be conserved by creating an additional defect whose moment is $-\varepsilon \psi_{\alpha} \hat{n}_{\alpha}$, which we see immediately is a dipole or tear defect.

A fold defect thus sheds tear defects to compensate its moment change when displaced in the "wrong" direction. Figure 3 shows the configuration of the plate obtained by transporting a fold defect with strength $-\delta_{\alpha 2}$ from $x_{2}=-\infty$ along the $x_{2}$ axis to the origin, shedding a uniform density of tear defects along its path: the lineon tears the plate in the process. (This configuration satisfies Eq. (17) precisely as the fold defect shown in Fig. 2(b) and both have identical curvature distributions.)

We hasten to point out that, while the duality provides insights into commonly observed phenomena related to folding and tearing, it does not take into account the "nonlinear" irreversible or plastic processes that accompany the motion of defects (treatment similar to Ref. [24]).

Indeed, the crumpling of paper [36-38], governed by the interplay of out of plane deformation and in-plane stretching, is not considered at all in the linear theory presented

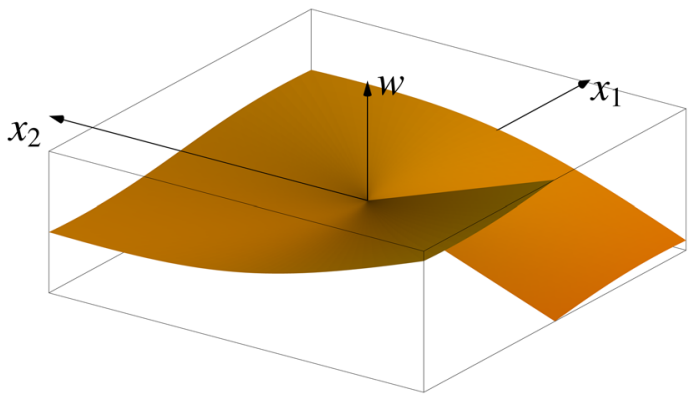

FIG. 3. Configuration obtained by transporting a fold defect with strength $\psi_{\alpha}=-\delta_{\alpha 2}$ from $x_{2}=-\infty$ along the $x_{2}$ axis to the origin. Displacement $w\left(x_{1}, x_{2}\right)=(1 / 2 \pi)\left[x_{1} \ln (|x|)+\right.$ $\left.x_{2} \arctan \left(x_{1} / x_{2}\right)\right]$ satisfies Eq. (17) and its curvatures are identical to Fig. 2(b). here. The structures that arise in crumpled paper attempt to minimize the in-plane stretching energy maximizing regions where Gaussian curvature vanishes. It will be interesting to explore generalizations of our formulation to situations where in plane deformation and out of plane deformation are coupled (Flöppl-von Karman theory [32,33]).

We conclude by noting that our work provides an example of a physical system with a dual embodiment of fractonic physics, adding to a growing list [24,39-43]. It will be interesting to explore other systems to find further examples. The general structure of fracton theories discussed in Ref. [17] might provide clues to look for the dual physical realizations.

N. M. acknowledges the KVPY programme, and V. B. S. thanks SERB, DST for support. This work was in part supported by the Deutsche Forschungsgemeinschaft under SFB 1143 (project-id 247310070) and cluster of excellence ct.qmat (EXC 2147, project-id 390858490).

nandagopalm@iisc.ac.in moessner@pks.mpg.de shenoy@iisc.ac.in

[1] A. Kitaev, Ann. Phys. (Amsterdam) 303, 2 (2003).

[2] C. Chamon, Phys. Rev. Lett. 94, 040402 (2005).

[3] S. Bravyi, B. Leemhuis, and B. M. Terhal, Ann. Phys. (Amsterdam) 326, 839 (2011).

[4] C. Castelnovo and C. Chamon, Philos. Mag. 92, 304 (2012).

[5] J. Haah, Phys. Rev. A 83, 042330 (2011).

[6] B. Yoshida, Phys. Rev. B 88, 125122 (2013).

[7] S. Bravyi and J. Haah, Phys. Rev. Lett. 111, 200501 (2013).

[8] S. Vijay, J. Haah, and L. Fu, Phys. Rev. B 92, 235136 (2015).

[9] S. Vijay, J. Haah, and L. Fu, Phys. Rev. B 94, 235157 (2016).

[10] D. J. Williamson, Phys. Rev. B 94, 155128 (2016).

[11] T. H. Hsieh and G. B. Halász, Phys. Rev. B 96, 165105 (2017).

[12] R. M. Nandkishore and M. Hermele, Annu. Rev. Condens. Matter Phys. 10, 295 (2019).

[13] M. Pretko, X. Chen, and Y. You, Int. J. Mod. Phys. A 35, 2030003 (2020).

[14] M. Pretko, Phys. Rev. B 95, 115139 (2017).

[15] M. Pretko, Phys. Rev. B 96, 035119 (2017).

[16] S. Pai and M. Pretko, Phys. Rev. B 97, 235102 (2018).

[17] V. B. Shenoy and R. Moessner, Phys. Rev. B 101, 085106 (2020).

[18] M. Pretko and L. Radzihovsky, Phys. Rev. Lett. 120, 195301 (2018).

[19] H. Kleinert, Gauge Fields in Condensed Matter (World Scientific, Singapore, 1989), Vol. 2.

[20] J. Zaanen, Z. Nussinov, and S. Mukhin, Ann. Phys. (Amsterdam) 310, 181 (2004).

[21] J. Dietel and H. Kleinert, Phys. Rev. B 73, 024113 (2006).

[22] A. J. Beekman, J. Nissinen, K. Wu, K. Liu, R.-J. Slager, Z. Nussinov, V. Cvetkovic, and J. Zaanen, Phys. Rep. 683, 1 (2017).

[23] A. Gromov, Phys. Rev. Lett. 122, 076403 (2019). 
[24] M. Pretko and L. Radzihovsky, Phys. Rev. Lett. 121, 235301 (2018).

[25] A. Kumar and A. C. Potter, Phys. Rev. B 100, 045119 (2019).

[26] Z. Zhai and L. Radzihovsky, Phys. Rev. B 100, 094105 (2019).

[27] M. Pretko, Z. Zhai, and L. Radzihovsky, Phys. Rev. B 100, 134113 (2019).

[28] A. Gromov and P. Surwka, SciPost Phys. 8, 65 (2020).

[29] D. Doshi and A. Gromov, arXiv:2005.03015.

[30] C. Xu, Phys. Rev. B 74, 224433 (2006).

[31] A. Rasmussen, Y.-Z. You, and C. Xu, arXiv:1601.08235.

[32] L. D. Landau and E. M. Lifshitz, Course of Theoretical Physics, Theory of Elasticity, 3rd ed. (Pergamon Press, Oxford, 1986), Vol. 7.

[33] E. H. Mansfield, The Bending and Stretching of Plates, 2nd ed. (Cambridge University Press, Cambridge, England, 1989).
[34] N. Seiberg, SciPost Phys. 8, 50 (2020).

[35] W. Shirley, K. Slagle, and X. Chen, Ann. Phys. (Amsterdam) 410, 167922 (2019).

[36] E. Cerda and L. Mahadevan, Phys. Rev. Lett. 80, 2358 (1998).

[37] T. A. Witten, Rev. Mod. Phys. 79, 643 (2007).

[38] O. Gottesman, J. Andrejevic, C. H. Rycroft, and S. M. Rubinstein, Commun. Phys. 1, 70 (2018).

[39] O. Benton, L. D. C. Jaubert, H. Yan, and N. Shannon, Nat. Commun. 7, 11572 (2016).

[40] Y. You and F. von Oppen, Phys. Rev. Research 1, 013011 (2019).

[41] S. R. Taylor, M. Schulz, F. Pollmann, and R. Moessner, Phys. Rev. B 102, 054206 (2020).

[42] V. Khemani, M. Hermele, and R. Nandkishore, Phys. Rev. B 101, 174204 (2020).

[43] J. Sous and M. Pretko, Quantum Mater. 5, 81 (2020). 